\title{
INVESTIGACIONES
}

\section{TIC y argumentación: Análisis de tareas propuestas por docentes universitarios*}

\author{
TIC and Argumentation: Analysis of Tasks Proposed by University Teachers
}

\section{TIC e argumentação: Análise tarefa proposta por acadêmicos}

\author{
José David Chávez Vescance, Adriana María Caicedo Tamayo. ${ }^{a}$ \\ ${ }^{a}$ Departamento de Ciencias Sociales, Pontificia Universidad Javeriana Cali. \\ Fono: 57-2-3218200-358. Correo electrónico: amc@javerianacali.edu.co
}

\begin{abstract}
RESUMEN
Aunque la argumentación es relevante para la construcción de conocimiento, los estudiantes encuentran dificultades para argumentar bien. No obstante, las TIC pueden utilizarse para mejorar las habilidades argumentativas. En esta investigación se analizaron 36 tareas apoyadas en TIC propuestas por docentes universitarios. Fueron analizados cuatro aspectos: ¿Las tareas demandaban argumentación? ¿Qué habilidades argumentativas exigían? ¿De qué manera las consignas estructuraban las demandas argumentativas? ¿Cómo se usaban las TIC en las tareas que demandaban argumentación? El análisis de las tareas reveló que fue poco frecuente la solicitud de evaluación de argumentos y el requerimiento de balanceo de pros y contras de posturas defendidas y refutadas. Además, se encontró que las plataformas virtuales de aprendizaje generalmente se utilizaron como repositorios de información y los tableros de discusión contaban con escasas restricciones para propiciar debates. A partir de la caracterización realizada, se propusieron pautas para diseñar tareas apoyadas en TIC que demanden argumentación.
\end{abstract}

Palabras clave: tecnología educacional, método de enseñanza, enseñanza superior.

\begin{abstract}
Although argumentation has an important place in knowledge building, students frequently find difficulties to build good arguments. However, ICT could be used to improve argumentative skills. This research analyzed 36 ICT supported tasks proposed by higher education teachers. The analysis took into consideration four questions: Did the task demand argumentation? Which argumentation skills were required? How were the argumentative demands structured? What kinds of uses were given to ICT in task that required argumentation? Task analysis revealed an unusual request of argument to the evaluation, as well as in the case of the requirement of pros and cons to defend or refute positions. Furthermore, we found that virtual learning environments were generally used as information repositories, and discussion boards had few restrictions to facilitate discussions. From these results, we propose guidelines for designing ICT supported tasks which demand argumentation.
\end{abstract}

Key words: educational technology, teaching methods, higher education.

\section{RESUMO}

Embora a argumentação seja relevante para a construção do conhecimento, os estudantes encontram dificuldades para bem argumentar. Não obstante, as TIC podem ser utilizadas para melhorar as habilidades argumentativas. Analisaram-se 36 tarefas apoiadas em TIC propostas por docentes universitários. Analisaram-se quatro aspectos:

* El presente artículo se deriva del proyecto "Tareas apoyadas en TIC y Argumentación”, financiado por Colciencias a través de la convocatoria Jóvenes Investigadores e Innovadores, en el marco del macroproyecto "Estrategias de Enseñanza apoyadas en TIC y construcción de conocimiento" financiado por la Pontificia Universidad Javeriana Cali (Código Proyecto 00003597) 
As tarefas pediam argumentação?, Quais habilidades argumentativas exigiam? De que maneira os slogans estruturavam os pedidos argumentativos? e Como se usavam as TIC nas tarefas que pediam argumentação? A análise das tarefas revelou a pouca frequência na aplicação de avaliação de argumentos e no requerimento de balanço de prós e contras de posturas defendidas e contraditas. Além do mais, encontraram-se as plataformas virtuais de aprendizagem que geralmente se utilizam como repositório de informações e os cartazes de discussão contavam com escassas restrições para propiciar debates. A partir da caracterização realizada, foram propostas pautas para desenhar tarefas apoiadas em TIC que pedem argumentação.

Palavras chave: tecnologia educacional, método de ensino, educação superior.

\section{INTRODUCCIÓN}

Cada vez es mayor el protagonismo de las Tecnologías de la Información y la Comunicación en la educación. Evidencia de lo anterior la constituyen los temas y objetivos que priorizan instituciones gubernamentales como el Ministerio de Educación Nacional de Colombia (MEN). En el Plan Nacional Decenal de Educación 2006-2016: Pacto social por la educación, por ejemplo, se establece que "debe garantizarse el acceso, uso y apropiación crítica de las TIC, como herramientas para el aprendizaje, la creatividad, el avance científico, tecnológico y cultural, que permitan el desarrollo humano y la participación activa en la sociedad del conocimiento" (Ministerio de Educación Nacional, 2007: 20). En el mismo documento, se plantea que resulta fundamental "la implementación de estrategias didácticas activas que faciliten el aprendizaje autónomo, colaborativo y el pensamiento crítico y creativo mediante el uso de las TIC" (Ministerio de Educación Nacional, 2007: 26).

La importancia que se atribuye al papel de las TIC en la educación se debe a algunas particularidades que las diferencian de cualquier otro medio de representación antes desarrollado por la humanidad. Para Martí (2003), existen siete características que particularizan a las nuevas tecnologías sobre cualquier otro tipo de herramienta. Dichas características son: formalismo, interactividad, dinamismo, multimedia, hipermedia, almacenamiento y transmisión. Cada una de estas particularidades tiene potencialidades educativas que a continuación se describen brevemente.

El formalismo alude a una forma singular de organizar y tratar la información: los programas de computador, aunque cada vez son más intuitivos y sencillos de utilizar, necesitan ser operados a través de secuencias lógicas y rígidas que el usuario debe asimilar, porque de lo contrario no es posible obtener eficientemente los resultados esperados. Dicha estructuración de los sistemas simbólicos y la manera singular de utilizarlos, según Coll (2004), exhorta la previsión y planificación de las acciones, así como la toma de conciencia y la autorregulación.

La interactividad hace referencia a la posibilidad de operar sobre la información y recibir una respuesta o retroalimentación inmediata. Las acciones de las personas transforman el estado de la información y la respuesta contingente, a su vez, orientan las posteriores actividades del usuario del ordenador. Esta relación recíproca entre la persona y el ordenador o la información brinda un papel activo al aprendiz, lo motiva y le permite controlar su ritmo de aprendizaje.

A la posibilidad de utilizar la información para construir representaciones de procesos se le denomina dinamismo. Fácilmente en un ordenador puede representarse el cambio o la evolución de los estados que experimentan distintos fenómenos, como el clima, la historia particular de un país o el movimiento de los astros. Gracias al carácter dinámico de las 
nuevas tecnologías es viable o factible la simulación de fenómenos, la anticipación del comportamiento de mecanismos o dispositivos de diversa complejidad. Esta cualidad de las TIC fomenta la experimentación, indagación y exploración, aspectos significativos para la enseñanza de las ciencias.

La riqueza que aporta el dinamismo se agranda exponencialmente cuando es considerada otra característica de las TIC: la multimedia. Con esto básicamente se hace alusión a la posibilidad de integrar o articular distintos formatos de representación en un mismo escenario digital. Un artículo de enciclopedia virtual, por ejemplo, puede tener grabaciones en audio y video, además de texto plano, gráficos y animaciones interactivas. Relacionarse con la información a través de diversas modalidades representacionales enriquece las elaboraciones mentales sobre los contenidos, facilitando la comprensión de los mismos y la generalización de los aprendizajes.

Toda esa información representada en múltiples formatos, a su vez, podría estar interconectada a través de complejas redes que abren "infinitos" caminos no lineales de exploración y presentación de los contenidos. A esta forma de organización no lineal de la información específicamente se le conoce como hipermedia. Esta característica, al igual que el dinamismo, incentiva la indagación, la exploración y el protagonismo del aprendiz en su proceso de aprendizaje.

Si el internauta puede navegar por océanos de textos, imágenes, videos y páginas web, es porque la capacidad de almacenar, recuperar y compartir gigantescas cantidades de información permanece en continuo crecimiento. A esto precisamente aluden las características denominadas transmisión y almacenamiento. Gracias a estas particularidades de las TIC es posible constituir ambientes virtuales de aprendizaje, comunidades educativas y escenarios que facilitan la colaboración en línea y la conformación de grupos y formas de trabajo antes insospechados.

Las ventajas o potencialidades educativas de las TIC, no obstante, están determinadas por el nivel de integración de las mismas a la enseñanza. Según Montes y Ochoa (2006), existen tres niveles de apropiación de las TIC a los procesos educativos: integración, reorientación y evolución. Cada uno de dichos niveles de apropiación influye singularmente en los procesos de enseñanza y aprendizaje y facilita resultados particulares. En el primer nivel de apropiación, llamado integración, las TIC se usan principalmente para comunicar y transmitir información efectivamente. En el segundo nivel, el de reorientación, las TIC sirven para proponer actividades que favorecen la construcción de conocimiento y el papel activo de los estudiantes. Por último, en el tercer nivel, el de evolución, los docentes ajustan, adaptan o transforman el uso convencional de las herramientas tecnológicas en la enseñanza, realizan innovaciones y divulgan sus avances en materia de utilización de las TIC para educar. El uso exclusivo de los espacios virtuales de aprendizaje para distribuir información acríticamente, sin proposición de tareas que den lugar a la controversia, el debate, la realización de simulaciones y experimentos, desaprovecha las múltiples herramientas para fomentar el desarrollo de distintos tipos de habilidades de razonamiento científico.

Una de esas habilidades que podría fomentarse a través de las TIC es la argumentación. Moore \& Marra (2005), por ejemplo, han planteado que el uso de las TIC es importante porque permite al aprendiz construir experiencias y conocimiento a partir del análisis de temas, posibilita la generación de cuestionamientos y el desafío de supuestos, facilita la realización de profundos insights, favorece la interacción grupal y potencia la adquisición de conocimientos. 
En el mismo orden de ideas, Evagorou \& Avraamidou (2008) han señalado que las TIC facilitan la búsqueda de evidencia, enriquecen la actividad reflexiva, reducen los obstáculos sociales y emocionales relacionados a la expresión de las propias opiniones e ideas y, por consiguiente, posibilitan que los estudiantes se involucren con más frecuencia e interés en las discusiones y actividades académicas (Brooks \& Jeong, 2006; Evagorou \& Avraamidou, 2008; Van Amelsvoort, Andriessen \& Kanselaar, 2007). De esta manera, herramientas como los foros virtuales, medios de comunicación asincrónica o no simultánea, brindan tiempo para la búsqueda de información y organización de las ideas, facilitan la utilización de múltiples formatos de representación, como imágenes, videos y grabaciones de audio, y además permiten obtener registros estables para monitorear el aprendizaje de los estudiantes (North, Coffin \& Hewings, 2008). Estos foros, según Brooks \& Jeong (2006), focalizan las intervenciones de los estudiantes en la discusión, evitan la reiteración de tópicos ya discutidos y disminuyen la composición de mensajes huérfanos - esos que no guardan relación alguna con los asuntos debatidos-.

Por lo menos dos razones justifican el interés de los investigadores en las TIC y su potencial para fomentar el desarrollo de habilidades de argumentación. Una de las razones está asociada a los aspectos favorables o positivos de la argumentación: propicia la clarificación y organización del pensamiento, facilita la identificación y reparación de vacíos conceptuales, posibilita la solución de problemas complejos, brinda herramientas para el análisis riguroso de información, exige actividad cognitiva superior, promueve la construcción de conocimiento, y se constituye en una actividad fundamental para la enseñanza y ejercicio de las ciencias (Clark \& Sampson, 2007; De Vries, Lund \& Baker, 2002; Jonassen, 2010; Leitão, 2007).

La otra razón alude a los numerosos y frecuentes problemas de los estudiantes para argumentar bien. Se ha observado que éstos tienen dificultades para identificar posturas diferentes a la propia y rara vez exploran los pros y contras de perspectivas alternas o contrapuestas (Kuhn \& Crowell, 2011; Van Amelsvoort, Andriessen \& Kanselaar, 2007), apoyan sus ideas utilizando evidencia irrelevante (Evagorou \& Avraamidou, 2008) y evitan la confrontación, involucrándose superficialmente en la resolución de los problemas (De Vries, Lund \& Baker, 2002). Adicionalmente, los estudiantes formulan argumentos que carecen de coherencia y profundidad (Brooks \& Jeong, 2006), formulan conclusiones no coherentes con las premisas y, en raras ocasiones, evalúan las explicaciones elaboradas por otros (Clark \& Sampson, 2007).

La superación de tales debilidades no sólo es necesaria, sino también compleja, pues la composición de buenos argumentos involucra múltiples aspectos. Por ejemplo: la realización de actividades como la comunicación y evaluación de planteamientos; el uso de estrategias para resolver la oposición entre posturas y la justificación de proposiciones utilizando evidencia (Maloney \& Simon, 2006); el cumplimiento de criterios como la relevancia, la suficiencia y la aceptabilidad (Jonassen, 2010); y el desarrollo de habilidades para generar teorías causales, ofrecer evidencia, proponer explicaciones alternativas, refutar teorías alternativas e identificar condiciones que debilitarían las teorías propuestas o defendidas (Kuhn, 1999).

Si la argumentación tiene un papel fundamental en la construcción de conocimiento, la solución de problemas y el aprendizaje de las ciencias, y argumentar es una actividad compleja y exigente que los estudiantes realizan con notables dificultades, es claro que el desarrollo o mejoramiento de las habilidades de argumentación podría constituir un objetivo educativo de los docentes. Después de todo, en una sociedad donde el saber científico se actualiza rápida y continuamente, a los profesores no sólo les compete la enseñanza de contenidos académicos, sino también, la enseñanza de habilidades y estrategias para que los estudiantes sean excelentes aprendices durante toda su vida (Pozo y Monereo, 1999). Las 
TIC podrían utilizarse para constituir escenarios educativos que contribuyan a la realización de dicha misión, a saber, el desarrollo de habilidades superiores de pensamiento. Onrubia (2007) propone dos ideas que permitirían sustentar este planteamiento. Por un lado, cuando funcionan como herramientas de la mente, las TIC ofrecen ayudas que ningún otro tipo de recursos físicos puede dar: brindan instrumentos (como los simuladores, las bases de datos, las hojas de cálculo y las herramientas de representación visual) que potencializan y optimizan exponencialmente las limitadas capacidades de procesamiento de la mente. Por otro lado, al ser flexibles, o lo mismo, al ser sensibles al ritmo de aprendizaje del estudiante, pueden usarse de manera contingente de acuerdo a las necesidades educativas que el aprendiz experimenta momento a momento en su proceso de aprendizaje.

Indudablemente los docentes tienen la posibilidad de utilizar las particularidades y potencialidades educativas de las TIC para propiciar el desarrollo de habilidades de pensamiento científico como la argumentación. Como fue mencionado con anterioridad, el fomento de este tipo de habilidades no goza de poca relevancia, pues además de que los estudiantes con frecuencia tienen dificultades para argumentar válida, suficiente y pertinentemente, la argumentación juega un papel fundamental en la solución de problemas poco estructurados, el aprendizaje de las ciencias y la construcción de conocimiento. Ahora bien, si los maestros pueden utilizar el potencial educativo de las TIC para propiciar el desarrollo de habilidades de argumentación, cabe preguntarse si las tareas apoyadas en tecnología educacional que proponen están configuradas, constituidas o estructuradas para conseguirlo.

Por esta razón, en esta investigación se indagó cómo eran algunas tareas apoyadas en TIC propuestas por docentes universitarios, y de qué manera su diseño demandaba el desarrollo de habilidades de argumentación. Con el fin de responder a tales preguntas, se construyó una agenda de codificación que permitió explorar y describir las demandas argumentativas que hacían las tareas, la constitución de las consignas propuestas para estructurar dichas demandas, y los usos que los docentes hacían de las TIC en las tareas cuya resolución exigía elaboración de argumentos. Precisamente el análisis de estos aspectos y la exploración de la brecha entre lo posible y lo realizado cotidianamente, brindaron los insumos, datos o la información necesaria para la formulación de unas pautas para el diseño de tareas apoyadas en tecnología que propicien el desarrollo de habilidades de argumentación.

\section{MÉTODO}

\subsection{MUESTRA}

La muestra estuvo conformada por 36 tareas de 22 docentes universitarios. Las tareas consistían en documentos escritos que los docentes daban a sus estudiantes para asignarles actividades académicas articuladas al desarrollo del programa de la asignatura. Las tareas eran administradas a través de entornos virtuales de aprendizaje, como Blackboard o Moodle. De los 22 docentes que facilitaron sus tareas, uno pertenecía a la Facultad de Ciencias de la Salud, siete a la Facultad de Humanidades y Ciencias Sociales, siete a la Facultad de Economía, Administración y Contaduría y siete a la Facultad de Ingeniería, Arquitectura y Urbanismo. Solamente participaron en el estudio maestros que utilizaban plataformas virtuales de aprendizaje en sus cursos. Todos los maestros firmaron el consentimiento informado y contribuyeron voluntariamente en la investigación. 


\subsection{INSTRUMENTO}

Se elaboró una agenda de codificación (ver Tabla 1). La agenda contaba con tres categorías de análisis: (a) demanda argumentativa, (b) pautas de la consigna y argumentación y (c) uso de TIC y argumentación. La primera categoría permitía determinar dos aspectos: si la resolución de la tarea implicaba argumentación, y si era así, qué habilidad argumentativa era requerida (justificar posturas, evaluar evidencia, visualizar contraargumentos, refutar posiciones). La segunda categoría permitía identificar qué tipo de pautas brindaba la tarea para estructurar la demanda argumentativa. La tercera y última categoría servía para reconocer el rol de las TIC en tareas que demandaban argumentación. Cada categoría incluía diversos descriptores, organizados jerárquicamente e identificados con un código numérico. El supuesto que subyace a dicha organización jerárquica es que las tareas de argumentación varían según el nivel de la complejidad de la demanda argumentativa, la estructuración de las consignas y el uso de las TIC.

Tabla 1. Esquema de codificación para el análisis de tareas apoyadas en TIC en función de la argumentación

\begin{tabular}{|c|c|}
\hline Categoría & Descripción \\
\hline \multirow{10}{*}{$\begin{array}{c}\text { Demanda } \\
\text { argumentativa }\end{array}$} & Requerimiento de seguimiento y réplica de procedimientos. \\
\hline & $\begin{array}{l}\text { Requerimiento de contestación de preguntas cerradas. Resolución de la } \\
\text { tarea no demanda elaboración de argumentos. }\end{array}$ \\
\hline & $\begin{array}{l}\text { La tarea solicita el establecimiento de planteamientos. Requisición de } \\
\text { respuestas a preguntas abiertas que explícitamente no demandan } \\
\text { argumentación. }\end{array}$ \\
\hline & $\begin{array}{l}\text { La resolución de la tarea requiere la identificación o exploración de } \\
\text { posturas/teorías alternativas sobre un mismo asunto. }\end{array}$ \\
\hline & La tarea demanda contestación de preguntas cerradas de argumentación. \\
\hline & $\begin{array}{l}\text { Solicitud de establecimiento y sustentación de planteamientos a partir de } \\
\text { ejemplos, explicaciones. La sustentación puede realizarse a partir de } \\
\text { anécdotas, sentido común. }\end{array}$ \\
\hline & $\begin{array}{l}\text { Solicitud de establecimiento y sustentación de planteamientos consultando } \\
\text { bibliografía disciplinar u obteniendo evidencia. }\end{array}$ \\
\hline & $\begin{array}{l}\text { La tarea demanda evaluación de argumentos, los planteamientos y sus } \\
\text { sustentaciones. Se evalúan las ideas y la evidencia, su coherencia, } \\
\text { pertinencia, consistencia, suficiencia y veracidad. }\end{array}$ \\
\hline & $\begin{array}{l}\text { La resolución de la tarea requiere toma de postura y contraargumentación } \\
\text { de perspectiva opuesta. }\end{array}$ \\
\hline & $\begin{array}{l}\text { La tarea demanda balanceo de pros y contras de las posturas defendidas y } \\
\text { refutadas. }\end{array}$ \\
\hline \multirow{3}{*}{$\begin{array}{l}\text { Pautas de la } \\
\text { consigna y } \\
\text { argumentación }\end{array}$} & $\begin{array}{l}\text { La tarea carece de instrucciones/indicaciones para estructurar una/la } \\
\text { demanda argumentativa. }\end{array}$ \\
\hline & $\begin{array}{l}\text { La tarea incorpora indicaciones/pautas que estructuran, explícitamente, } \\
\text { una/la demanda argumentativa. }\end{array}$ \\
\hline & La tarea operacionaliza la demanda argumentativa. \\
\hline
\end{tabular}




\begin{tabular}{|c|c|}
\hline \multirow{8}{*}{$\begin{array}{l}\text { Usos de las TIC y } \\
\text { argumentación }\end{array}$} & Las TIC se utilizan para resolver una tarea que no demanda argumentación. \\
\hline & $\begin{array}{l}\text { Tarea de argumentación que implica utilización de las TIC para la gestión } \\
\text { de información. }\end{array}$ \\
\hline & $\begin{array}{l}\text { Las TIC se utilizan para administrar test/pruebas con preguntas cerradas de } \\
\text { argumentación. }\end{array}$ \\
\hline & $\begin{array}{l}\text { La tarea demanda argumentación. Las TIC apoyan el desarrollo de } \\
\text { productos. }\end{array}$ \\
\hline & $\begin{array}{l}\text { La solución de la tarea requiere utilización de TIC para el diseño de } \\
\text { experimentos, la realización de simulaciones y la planificación de métodos } \\
\text { de recolección de datos o evidencia. }\end{array}$ \\
\hline & $\begin{array}{l}\text { La resolución de la tarea implica utilización de TIC para asistir, } \\
\text { específicamente, la composición de argumentos. }\end{array}$ \\
\hline & $\begin{array}{l}\text { La resolución de la tarea implica utilización de las TIC para la confrontación, } \\
\text { análisis y evaluación de argumentos. }\end{array}$ \\
\hline & $\begin{array}{l}\text { Las TIC se utilizan para tutorar la resolución de tareas que demandan } \\
\text { argumentación. }\end{array}$ \\
\hline
\end{tabular}

Fuente: elaboración propia.

\subsection{PROCEDIMIENTO}

Se presentó la investigación a un grupo de docentes universitarios que utilizaban TIC en su enseñanza, y más específicamente, plataformas virtuales de aprendizaje como Blackboard y Moodle. De 183 maestros encuestados, 28 fueron seleccionados no-probabilísticamente. A estos docentes se les solicitó participar voluntariamente en el estudio. Después que firmaron el consentimiento informado, los docentes entregaron a los investigadores tareas apoyadas en TIC que implementaron en su enseñanza. Solamente se escogieron las tareas de 22 profesores, dado que los documentos de los demás docentes no aportaban suficiente información para llevar a cabo el análisis de contenido, o bien, no presentaban las tareas entregadas y resueltas por los estudiantes, sino descripciones generales sobre las mismas.

Una vez recogidas las tareas, se procedió a realizar el análisis de contenido utilizando una agenda de codificación. Para elaborar esta agenda, inicialmente se formularon unas categorías de análisis a partir de la revisión de la literatura (Cho \& Jonassen, 2002; Clark \& Sampson, 2007; Clark et al., 2007; Golanics \& Nussbaum, 2007; Kuhn \& Crowell, 2011; Simon, Erduran \& Osborne, 2006). Luego, dichas categorías se pilotearon y sometieron a valoración de jueces expertos. Posteriormente, estas categorías fueron utilizadas para realizar un análisis preliminar de las tareas del estudio. A partir de este análisis preliminar, se identificaron e incluyeron descriptores de las tareas que no fueron inicialmente contemplados. Una vez construida la versión final del instrumento, se procedió a realizar el análisis de contenido de todas las tareas recogidas.

\subsection{ANÁLISIS DE LOS DATOS}

Las tareas fueron examinadas utilizando la técnica de análisis de contenido propuesta por Philipp Mayring (Kohlbacher, 2006; Mayring, 2004, 2002). Todo el texto de las tareas fue objeto de análisis y cada tarea recibía solamente un código por categoría. Por cada código asignado, se describía el rasgo de la tarea asociado al código y la justificación o 
explicación de la codificación atribuida. Un evaluador externo revisó las codificaciones asignadas por el investigador principal. Los desacuerdos fueron discutidos y se reasignaron nuevos códigos a las codificaciones controvertidas. Posteriormente, se reagruparon las tareas de acuerdo a los códigos atribuidos en cada categoría. Luego, para las cuatro categorías de análisis, se examinaron e interpretaron las descripciones y justificaciones agrupadas en los mismos códigos (por ejemplo: “¿Qué caracterizaba a todas las tareas cuya demanda era contestación de preguntas cerradas? ¿Qué caracterizaba a las tareas que carecían de pautas para estructurar la demanda argumentativa?"). Finalmente, se obtuvieron frecuencias absolutas y relativas de los códigos asignados.

\section{RESULTADOS}

Se analizó el contenido de 36 tareas de docentes universitarios que implementaron TIC en su enseñanza. Se exploraron tres aspectos: (a) la demanda argumentativa de las tareas, (b) las pautas de la consigna para estructurar la demanda argumentativa, y (c) el uso de las TIC. A continuación, se mencionan los principales hallazgos observados en cada uno de estos aspectos (ver Tabla 2).

Tabla 2. Frecuencia absoluta y relativa de las tareas encontradas en función del análisis de la demanda argumentativa, las pautas de las consignas y el uso de las TIC en tareas de argumentación

\begin{tabular}{|c|c|c|}
\hline Categorías de análisis y descriptores & $f$ & $\%$ \\
\hline \multicolumn{3}{|l|}{ Demanda argumentativa } \\
\hline Preguntas cerradas sin demanda argumentativa. & 5 & 13.9 \\
\hline Preguntas abiertas sin demanda argumentativa. & 7 & 19.4 \\
\hline Pedido de argumentación sin requerimiento de evidencia. & 1 & 2.8 \\
\hline $\begin{array}{l}\text { Solicitud de argumentación a partir de evidencia, bibliografía, } \\
\text { experimentación o simulaciones. }\end{array}$ & 19 & 52.8 \\
\hline Demanda de evaluación de argumentos y evidencia. & 2 & 5.6 \\
\hline $\begin{array}{l}\text { Requerimiento de balanceo de pros y contras de posturas defendidas y } \\
\text { refutadas. }\end{array}$ & 2 & 5.6 \\
\hline \multicolumn{3}{|l|}{ Pautas de la consigna y argumentación } \\
\hline Ausencia de pautas para la estructuración de la demanda argumentativa. & 24 & 66.7 \\
\hline Consignas con pautas para la estructuración de la demanda argumentativa. & 9 & 25.0 \\
\hline Pautas con operacionalización de la demanda argumentativa. & 3 & 8.3 \\
\hline \multicolumn{3}{|l|}{ Uso de las TIC y argumentación } \\
\hline TIC en tareas sin demanda argumentativa. & 12 & 33.3 \\
\hline TIC para la gestión de información en tareas de argumentación. & 22 & 61.1 \\
\hline TIC para el apoyo de confrontación, análisis y evaluación de argumentos. & 1 & 2.8 \\
\hline TIC para tutoría de tarea de argumentación. & 1 & 2.8 \\
\hline
\end{tabular}

Fuente: elaboración propia. 


\subsection{LA DEMANDA ARGUMENTATIVA}

Para empezar, se identificaron cuáles eran las tareas cuya resolución requería la elaboración de argumentos. Del total de tareas, el 67\% demandaba argumentación. El nivel de complejidad de las demandas variaba de tarea en tarea, es decir, algunas consignas requerían realización de actividades más elaboradas que otras: del $67 \%$ de tareas que requería argumentación, el $63.9 \%$ de las tareas pedían composición de argumentos a partir de evidencia, contenidos académicos estudiados previamente o revisión de bibliografía disciplinar, mientras el otro $2.8 \%$ de las tareas requería argumentación desde el conocimiento previo o las creencias de los estudiantes.

Del $63.9 \%$ de tareas que requería composición de argumentos a partir de evidencia, simulaciones o revisión de literatura científica, el 5.6\% demandó la evaluación de argumentos en función de su coherencia, consistencia, suficiencia, validez o veracidad, y otro 5.6\% requirió la confrontación y balanceo de pros y contras de posturas defendidas y refutadas. Para propiciar la evaluación de argumentos, algunas tareas solicitaban la escritura de textos argumentativos que debían ser publicados en un tablero de discusión para que otro compañero hiciera sus críticas argumentadas. Por otra parte, para favorecer el balanceo de aspectos favorables y desfavorables de las posturas defendidas y refutadas, podía pedirse la contestación individual y sustentada de solicitudes como la siguiente: “¿Es posible el desarrollo sostenible para América Latina? Expliquen si el desarrollo es posible o no y cuáles son sus principales obstáculos y/o potencialidades” (Tarea 12).

Ahora bien, la demanda argumentativa de las tareas en ocasiones fue implícita. Esto se observó en el $41.7 \%$ de las tareas que demandaron argumentación. La Tarea 36, por ejemplo, pedía a los estudiantes que, a partir de tres temáticas (moral cristiana, pluralismo cultural y ética de máximos y mínimos) elaboraran un escrito donde se mencionara la relación entre máximos y mínimos en una sociedad pluralista. El escrito debía cumplir con ciertos criterios de evaluación, como la utilización de los textos leídos. La consigna explícitamente no requería la justificación, explicación o sustentación de la respuesta, no obstante, el análisis de la tarea revelaba que para resolver la misma no bastaba con enunciar la relación entre los conceptos en cuestión, sino que debía explicarse, con bibliografía, la respuesta formulada.

Otras tareas cuya demanda argumentativa también tuvo que inferirse, consistían en lineamientos o pautas generales para el desarrollo de proyectos. Éstos requerían la elaboración de múltiples subproductos que debían sustentarse durante y después de la finalización del proyecto, sin que se presentaran pautas sobre la naturaleza de tales actividades.

Por otra parte, una menor proporción de tareas (33\%) no demandaba argumentación. Este tipo de tareas, por lo general, solicitaba la búsqueda y exposición de información, la realización de descripciones, la recuperación de información específica o la resolución de ejercicios para repasar contenidos vistos previamente. Una ilustración de este tipo de tareas eran los test cortos virtuales que requerían la contestación de preguntas cerradas para evaluar las temáticas vistas o para realizar controles de lectura, en estos casos, los estudiantes tenían que determinar si una proposición era falsa o verdadera, sin explicitar la explicación de su decisión.

En el análisis de contenido de las tareas, también se observó que no toda tarea que explícitamente demandaba explicaciones era de argumentación. Había interrogantes que 
a pesar de pedir explicaciones, podían contestarse a través de descripciones. Obsérvese como ejemplo el caso de las siguientes tareas: una tarea asignaba textos de lectura sobre tipos de entrevista y posteriormente solicitaba la realización de un taller sobre las lecturas consultadas. Una de las preguntas del taller decía: "Explique brevemente, en relación con la entrevista: la entrevista dirigida y la entrevista no dirigida". Nótese que para responder al requerimiento bastaba con ofrecer descripciones o definiciones sobre ambos conceptos y no era necesario explicar (Tarea 8). Algo similar sucedía cuando se requería la explicación de gráficos elaborados a partir de datos recogidos. En este caso, la tarea no brindaba pautas para diferenciar descripciones de explicaciones, de tal modo que la tarea podía resolverse mencionando características de la figura cuando se estaba pidiendo inferir y justificar causas o consecuencias de la imagen obtenida.

El análisis también permitió observar que una tarea macro podía estar constituida por múltiples sub-tareas; mientras sólo una demandaba argumentación, las otras requerían descripciones, definiciones o información puntual y específica como "¿Qué es un accidente de trabajo? ¿Qué son horas hombre trabajadas?” (Tarea 14).

\subsection{PAUTAS DE LAS CONSIGNAS Y ARGUMENTACIÓN}

Las tareas que demandaban argumentación requerían la realización de diversos tipos de actividades, como por ejemplo: (a) establecer un planteamiento y solicitar a los estudiantes toma y justificación de postura; (b) requerir la identificación de conceptos en un caso (presentado en texto plano o a través de videos) y la explicación de la asociación entre el concepto y el ejemplo hallado; (c) la escritura de un ensayo que respondiera a una pregunta dada -la resolución de la actividad exigía el planteamiento de la respuesta y la sustentación de la misma a través de argumentos-; (d) análisis de casos (reales, diseñados por estudiantes o maestros) estableciendo valoraciones, diagnósticos y propuestas de acción-intervención amparadas en teoría disciplinar o contenidos vistos; (e) desarrollo de productos (software, microprocesadores, propuestas de intervención) y sustentación de los mismos, y; (f) obtención de datos para la realización de gráficas que debían ser analizadas y explicadas.

El 50\% de las tareas que demandaban argumentación brindaba algún tipo de pauta o indicación para estructurar la demanda argumentativa. Algunas pautas eran más estructuradas que otras. El mayor grado de estructuración se observaba cuando la tarea operacionalizaba la demanda que hacía: cuando se explicitaba cómo elaborar los argumentos, en qué consistía justificar, explicar o sustentar. Este tipo de demanda se observó en el $12 \%$ de las tareas de argumentación. En estos casos, las tareas utilizaban ejemplos o definían los verbos que determinaban la actividad a realizar. Por ejemplo, para el verbo "analizar", la tarea explicitaba "distinción y separación de las partes de un todo hasta llegar a conocer sus principios o elementos" (Tarea 15). El otro 38\% de las tareas de argumentación no operacionalizaba la demanda argumentativa, pero indicaba qué recursos utilizar (videos, textos), qué métodos emplear (“demuestre con sus propias palabras que...”, Tarea 6), qué criterios debían cumplir los argumentos ("deben estar relacionados con la tesis... la tesis está formulada con claridad... los argumentos tienen una justificación desde los fundamentos económicos... el conjunto de argumentos es suficiente para que el autor adopte una postura frente a la tesis", Tarea 24) y en qué información tenían que hacer énfasis ("potencialidades y obstáculos", Tarea 12). 
La otra mitad de tareas de argumentación carecía de indicaciones para precisar a los estudiantes cómo debían constituirse o elaborarse los argumentos. No había pautas que explicaran al estudiante métodos, recursos o criterios para elaborar sus productos y sustentaciones cuando la demanda argumentativa de la tarea era implícita.

\subsection{USO DE LAS TIC Y ARGUMENTACIÓN}

Del total de tareas que demandaban argumentación (66.7\%), el 61.1\% utilizó las plataformas virtuales de aprendizaje para la gestión de información, es decir, para buscar, presentar, enviar, publicar y recibir información, documentos, imágenes y archivos. Por otra parte, en el $5.6 \%$ de tareas restantes que demandan elaboración de argumentos (dos tareas en total), las plataformas virtuales de aprendizaje estuvieron integradas a la clase y constituyeron escenarios para facilitar el desarrollo de procesos educativos. Una de estas tareas requería el uso de tableros de discusión para que los estudiantes asumieran el rol de evaluadores y retroalimentaran críticamente los ensayos escritos por sus compañeros (Tarea 25). La otra tarea requirió la realización de diferentes actividades, como la utilización de un tablero de discusión para el análisis de un cuento a partir de unas lecturas previas, y para la escritura, distribución y análisis colectivo de casos (Tarea 20).

Del total de tareas analizadas, el 5.6\% utilizaba tableros de discusión y demandaban argumentación. En el $94.4 \%$ de tareas restantes también se implementaron foros, pero las consignas de trabajo no propiciaban debate o argumentación. En estos casos los foros se utilizaban para solicitar la exposición de puntos de vista que no necesariamente debían entrar en confrontación. Solamente una tarea estuvo estructurada para fomentar argumentación e implementar tableros de discusión (Tarea 24). En esta tarea cada estudiante tenía que escribir un texto a partir de una pregunta dada que exigía la toma de postura: “Las organizaciones colombianas están preparadas para enfrentarse a los desafíos de un entorno globalizado, cambiante, innovador y de alto nivel de competitividad?". Posteriormente, a cada estudiante se le asignaba un par evaluador. Los ensayos tenían que publicarse en un tablero de discusión para ser evaluados por los pares escogidos. La idea es que pudiera suscitarse un debate entre los pares a partir de las evaluaciones que recíprocamente hacían de los trabajos elaborados.

Las TIC también fueron implementadas para administrar evaluaciones. No obstante, dichas pruebas estaban constituidas por preguntas cerradas que, como se mencionó anteriormente, no demandaban argumentación.

\section{DISCUSIÓN}

Con el fin de explorar cuáles son los usos dados a las TIC en la educación superior, se propuso caracterizar 36 tareas diseñadas y propuestas por docentes universitarios. Esta caracterización hizo énfasis en la argumentación, y más específicamente, en tres aspectos: la demanda argumentativa de las tareas, las pautas que estructuraban la demanda argumentativa, y el uso de las TIC en tareas cuya resolución requería argumentación.

Los principales resultados obtenidos a partir del análisis de contenido de las tareas muestran que la mayoría de éstas demandaban argumentación a través del análisis de evidencia o de la consulta de bibliografía académica. Adicionalmente, se encontró que 
fueron pocas las tareas que solicitaban la evaluación de argumentos (los planteamientos y sus respectivos soportes) o la confrontación y balanceo de pros y contras de posturas defendidas y refutadas. También se observó que la mitad de tareas analizadas brindaba indicaciones para estructurar las demandas argumentativas, pero pocas operacionalizaron en qué consistía la demanda argumentativa. Además de lo anterior, pudo apreciarse que las TIC generalmente se usaron como repositorios de información y los tableros virtuales de discusión (foros) no contaron con consignas que, precisamente, propiciaran el debate. En lo que sigue, estos hallazgos serán discutidos con más detalle.

Al diseñar tareas de argumentación, con frecuencia los docentes piden a sus estudiantes elaborar explicaciones esperando que ellos construyan argumentos para responder a la tarea. Si bien la argumentación es el proceso de coordinación e interconexión de planteamientos, evidencia y explicaciones para apoyar o contradecir una postura (Cho \& Jonassen, 2002; Evagorou \& Avraamidou, 2008; Jeong, 2006; Leitão, 2001, 2000; Moore \& Marra, 2005; Simon, Erduran \& Osborne, 2006; Stark, Puhl \& Krause, 2009), las pautas propuestas por los docentes no siempre hacen este tipo de demandas explícitamente, sino que proponen consignas que demandan procesos de descripción o selección de información. Si haciendo descripciones se resuelve efectivamente una tarea que explícitamente requiere argumentación, entonces posiblemente la consigna está enunciando el verbo inapropiado.

En esta investigación se encontró que la mayoría de tareas analizadas solicitaba la composición de argumentos utilizando bibliografía académica u obteniendo evidencia. Al menos desde el análisis de contenido, se percibe que los maestros pretendían que sus estudiantes sustentaran sus ideas a partir de los conocimientos disciplinares abordados. Esto resulta llamativo porque los estudios a menudo reportan que los estudiantes con frecuencia argumentan sin aportar o utilizar adecuadamente la evidencia (Scheuer et al., 2010; Stark, Puhl \& Krause, 2009). Aunque asegurar lo anterior requeriría observar el desempeño de los estudiantes, por lo menos a partir del análisis realizado es posible afirmar que las tareas explícitamente demandan argumentación utilizando bibliografía académica u obteniendo evidencia o soportes.

Ahora bien, conviene preguntarse de qué maneras se hicieron estas demandas argumentativas. Lo mínimo que debe requerir una tarea de argumentación es la toma y justificación de una postura a través de la utilización de fundamentos, provengan éstos de la propia experiencia, o de la recolección sistemática de información. Esta condición básica fue observada en la mayoría de tareas analizadas. Se encontró que los maestros solicitaron toma y justificación de posturas de múltiples maneras: a partir de análisis de casos, aplicación de conceptos, desarrollo de productos, contestación a preguntas dicotómicas, elaboración de ensayos argumentativos y análisis de gráficas de acuerdo a datos acopiados, entre otras. Las distintas y variadas técnicas observadas indican que, cuando se trata del diseño de tareas que demanden argumentación, los maestros tienen a su disposición una extensa gama de posibilidades (para conocer otras alternativas en el área de ciencias, se recomienda revisar los trabajos de Sampson \& Gerbino, 2010; Simon \& Maloney, 2007; Simon \& Richardson, 2009).

Probablemente, de los objetivos educativos del maestro, explícitos o no, depende la proposición de actividades que promuevan el desarrollo de habilidades para argumentar. Si, como se mencionó con anterioridad, la argumentación tiene un rol importante en la construcción de conocimiento y la solución de problemas complejos, y además es posible mejorar las habilidades de argumentación (Kuhn, et al., 2008; Kuhn \& Udell, 2003), entonces no resulta 
trivial explorar qué concepciones del maestro prevalecen sobre el aprendizaje y la enseñanza y cómo dichas concepciones contribuyen, no solamente a la apropiación de contenidos, sino también al desarrollo de habilidades de pensamiento superior (Pozo et al., 2006).

Por otra parte, demandas como la evaluación de argumentos y el balanceo de pros y contras de posturas defendidas y refutadas fueron encontradas con menos frecuencia. Evaluar la evidencia, datos, soportes y su articulación con los planteamientos es una habilidad necesaria para la composición de argumentos válidos, veraces y consistentes (Evagorou \& Avraamidou, 2008). Sin embargo, ésta fue una demanda que se observó con poca frecuencia, en tanto que los estudiantes no eran impelidos a evaluar las argumentaciones, propias o de otras personas. Precisamente, uno de los principales problemas que enfrentan los estudiantes al argumentar es la selección y utilización pertinente y suficiente de evidencia, es por esto que los docentes cuyo propósito sea mejorar la articulación entre evidencia y planteamientos se beneficiarían de actividades dirigidas a la evaluación de los argumentos. De hecho, la evaluación de la evidencia es, según Evagorou \& Avraamidou (2008), una actividad necesaria para la argumentación y la enseñanza de las ciencias.

De manera similar, los estudiantes generalmente tienen dificultades para balancear los pros y contras de las perspectivas defendidas y refutadas; perciben más fácilmente las fortalezas de las posturas defendidas y las debilidades de los puntos de vista criticados, que los inconvenientes de las teorías propugnadas y las ventajas de las teorías refutadas. Además de esto, las personas tienden a ignorar o desvalorizar la evidencia que no apoya sus ideas (Kuhn \& Crowell, 2011; Scheuer et al., 2010; Van Amelsvoort, Andriessen \& Kanselaar, 2007). Facilitar la consideración de las fortalezas y debilidades de las ideas defendidas y criticadas posibilita la composición de argumentos integradores, consistentes y difícilmente rebatibles. A pesar de esto, pocas de las tareas analizadas estuvieron diseñadas para demandar el balanceo de pros y contras de las posturas defendidas y refutadas. Por consiguiente, es necesario propiciar o crear pautas para el diseño de tareas de argumentación apoyadas en TIC que demanden balanceo de posturas. Por ejemplo, Stegmann, Weinberger $\&$ Fischer (2007) encontraron que utilizando formatos para estructurar el modo o el tipo de publicaciones que hacen los estudiantes se mejora la calidad estructural de los argumentos, así como la generación de contraargumentos.

Una herramienta frecuentemente citada en la literatura por su potencial para diseñar tareas de argumentación son los tableros de discusión, en tanto se constituyen en espacios virtuales idóneos para el debate (Moore \& Marra, 2005; North, Coffin \& Hewings, 2008). No obstante, en este estudio se encontró que pocas tareas implementaban esta herramienta, y cuando lo hacían, las tareas analizadas generalmente no disponían de actividades y consignas que propusieran la toma y confrontación de posturas. Para resolver las tareas, los estudiantes podían aportar ideas que espontáneamente entraran en contradicción con las opiniones de otras personas. Así pues, si el docente desea que el tablero de discusión fomente el debate, necesita diseñar consignas con restricciones que garanticen la toma, confrontación y justificación de posturas; no basta la solicitud de opiniones generales sobre asuntos que no polarizan las ideas. En la literatura se reportan múltiples estrategias para diseñar tareas con tales características. Clark \& Sampson (2007), por ejemplo, pidieron a estudiantes de octavo grado que recogieran datos y luego formularan un principio a partir de la evidencia. Posteriormente, propiciaron la confrontación de las posturas de estudiantes que propusieron principios diferentes a través de un tablero de discusión, que priorizó el análisis y la crítica de perspectivas contrapuestas. 
Ciertamente, para diseñar tareas apoyadas en TIC que fomenten argumentación, no basta con seleccionar una herramienta tecnológica apropiada, sino que también es necesario plantear consignas pertinentes, claras y completas. Consignas de este tipo fueron observadas en la mitad de las tareas de argumentación analizadas. En estos casos las tareas brindaban pautas o indicaciones para estructurar la demanda argumentativa, señalaban criterios, recursos y metodologías que los estudiantes podían seguir o implementar.

El análisis de este tipo de tareas hizo posible identificar algunas recomendaciones orientadas a la composición de consignas más estructuradas. Por ejemplo, si la tarea pide a los estudiantes que los argumentos sean "coherentes", "consistentes", "suficientes", "relevantes" y "veraces", éstos necesitan saber qué significa cada criterio. Por otra parte, si la tarea solicita sustentación de las respuestas, es oportuno explicitar, a través de la definición de verbos o ilustración con ejemplos, de qué manera se escriben y constituyen los argumentos, cómo se analizan, evalúan y refutan. Un profesor, por ejemplo, que pretenda solicitar elaboración de justificaciones para explorar qué tanto comprenden un concepto sus estudiantes, podría proponer lo siguiente: “identifique ejemplos del concepto X en el caso $\mathrm{Z}$ y justifique su respuesta. Para justificar, mencione el ejemplo, formule una tesis que relacione el concepto con el caso y explique utilizando los textos leídos con anticipación, por qué es válida la relación que usted propone, esto es, por qué es coherente la evidencia que usted utiliza para sustentar la tesis que plantea".

Justamente, consignas con tales características son sugeridas por autores que investigan el aprendizaje autorregulado. Desde esta perspectiva, se plantea que las tareas deben ofrecer a los estudiantes consignas y objetivos de tareas claros que les permitan formularse estándares o metas propias para que planifiquen, monitoreen y controlen sus procesos de resolución de tareas (Ley \& Young, 2001; McMahon \& Oliver, 2001; Perry \& Winne, 2006; Valencia, Duarte y Caicedo, 2011; Winne, 2001; Winne \& Hadwin, 1998; Winters, Greene \& Costich, 2008). Dicho de otro modo: la adecuada estructuración de las pautas permite que los estudiantes desplieguen las estrategias de aprendizaje necesarias para alcanzar los propios estándares y los objetivos educativos propuestos por el maestro.

A pesar de lo anterior, es necesario hacer una salvedad. La escritura de consignas y objetivos claros y completos, donde se operacionalice la demanda de la tarea, no es una actividad sencilla. Explicitar, por ejemplo, en qué consiste "justificar", "analizar" y "explicar" un desarrollo, respuesta o producto exige que el docente describa las operaciones o acciones, incluso mentales, que debe realizar el estudiante. Si el docente tiene dificultades para detallar qué acciones implican las demandas que realiza, muchos más obstáculos encontrará el estudiante para formularse unas metas coherentes con los propósitos educativos del maestro.

Ahora bien, con relación al uso de las TIC y su aporte al diseño de escenarios potencialmente educativos, se encontró que las tareas analizadas generalmente utilizaban las tecnologías como repositorios de información. En los entornos virtuales de aprendizaje se alojaban los materiales de trabajo que los estudiantes debían descargar, guardar y realizar. Las evaluaciones administradas a través de plataformas como Blackboard estaban constituidas por preguntas cerradas cuya resolución implicaba, por lo general, recuperación y evocación de los contenidos estudiados. Rara vez una pregunta cerrada presentada en evaluaciones virtuales demandaba comprensión, análisis, argumentación o proposición. Aunque la resolución de las tareas generalmente requería la búsqueda de información en internet o bases de datos, el uso de procesadores de texto, la utilización de diferentes 
programas para realizar cálculos, simulaciones y obtener evidencia, la principal actividad de enseñanza mediada por los ambientes virtuales de aprendizaje era la distribución de contenidos del currículo. Dicho en otros términos: los estudiantes sí tenían que utilizar herramientas tecnológicas para resolver las tareas propuestas por los docentes -resultaba prácticamente imposible no hacerlo-, pero como recursos para constituir escenarios auténticos de aprendizaje que mediaran las relaciones, interacciones y comunicaciones entre compañeros, tutores y contenidos, las TIC no jugaron un papel relevante que trascendiera la asignación de lecturas y actividades y el envío de archivos, páginas o documentos y, por consiguiente, los principales intercambios educativos se fomentaron, al menos formalmente, fuera de la virtualidad.

Este tipo de uso de las TIC es básico, según los tres niveles de apropiación propuestos por Montes y Ochoa (2006) para analizar la utilización de las herramientas tecnológicas en las prácticas educativas de docentes universitarios. Un asunto que posiblemente explica el bajo nivel de integración de las TIC a las actividades educativas es la modalidad de enseñanza impartida en las asignaturas de las tareas analizadas. Casi todos los cursos tenían una modalidad de enseñanza presencial. Cuando la mayoría de actividades de enseñanza tienen la posibilidad de ejecutarse efectivamente en el aula de clase (discusiones, comprobaciones de lectura, exposición de contenidos, resolución colaborativa de problemas, supervisión de proyectos), la utilización de las TIC para configurar otros escenarios educativos se transforma en un asunto opcional, o lo mismo, no indispensable (Jonassen, 2010).

Tal situación, por supuesto, no es la ideal, pues las TIC ofrecen ventajas o particularidades que podrían aprovecharse para fomentar el desarrollo de habilidades de argumentación (Coll, 2004; Martí, 2003; Munneke et al., 2007; Munneke, Van Amelsvoort \& Andriessen, 2003; Onrubia, 2007). Las herramientas de representación visual (como Belvedere, DebateGraph, SenseMaker), por ejemplo, posibilitan y exigen organizar y explicitar el pensamiento o las ideas, hacen evidente los argumentos y contraargumentos, sus fortalezas y debilidades, sus componentes y relaciones. Los tableros virtuales de discusión, por otro lado, brindan al docente rastros o huellas de los estados de comprensión de los estudiantes sobre las temáticas en debate, reducen la amenaza o miedo social que muchas personas sienten a expresar sus opiniones, facilitan la apertura de espacios para la reflexión y la elaboración de las ideas, y admiten la utilización de múltiples formatos de representación (texto, gráficos, video, audio, animaciones).

Evidentemente, toda tarea apoyada en TIC que demande argumentación podría usar a su favor las cualidades que singularizan a los recursos tecnológicos sobre cualquier otra herramienta jamás creada: el dinamismo y la interactividad facilitarían la realización de simulaciones, cálculos y experimentos para comprobar hipótesis u obtener, evaluar y utilizar evidencia; los múltiples formatos de representación (multimedia) podrían aprovecharse para construir argumentos fuertes y suficientemente sustentados con distintos tipos de información; la red de redes contribuiría a que la pesquisa de esos fundamentos sea una auténtica navegación por extensos y ramificados caminos de información (hipermedia y almacenamiento); y la conectividad y las facilidades de transmisión de información harían que tal viaje no fuera una aventura solitaria, pues los tutores y los pares siempre podrían estar allí, bien para apoyar la búsqueda y la construcción de los argumentos, bien para evaluar, contraargumentar o refutar los planteamientos presentados.

Dado que la mayoría de tareas analizadas reflejó un bajo nivel de apropiación de las TIC, y existen niveles de apropiación más elevados que otros, parece claro que aún puede 
aprovecharse más el potencial educativo de las herramientas tecnológicas para el diseño de tareas que propicien argumentación. No obstante, es preciso tener en consideración lo siguiente: la principal fuente de información fueron los documentos escritos, y más específicamente, las consignas de trabajo que los docentes daban a sus estudiantes. Como las tareas analizadas hacían parte en su mayoría de asignaturas cuya modalidad de enseñanza era presencial, es probable que durante las clases se dieran especificaciones o aclaraciones que no aparecían en las consignas escritas. Evidentemente, se requiere la utilización y el análisis de otras fuentes de información (como entrevistas) para triangular los datos recogidos, y obtener una visión más completa del fenómeno estudiado.

Aun así, el análisis de las tareas con los insumos recogidos aportó evidencia e ideas para el diseño de pautas o indicaciones para la creación de tareas apoyadas en TIC que demanden argumentación. En un estudio posterior se evaluaría el desempeño argumentativo de estudiantes que enfrentaran tareas diseñadas a partir de dichas pautas. Por otra parte, las categorías de análisis desarrolladas en este estudio también tienen potenciales aplicaciones educativas, puesto que podrían considerarse o implementarse como recurso guía para el diseño de tareas cuyo propósito sea desarrollar habilidades para argumentar. Precisamente, cada uno de los descriptores del instrumento brinda al docente indicios para anticipar el nivel de complejidad de la demanda argumentativa que su tarea pide o pediría. En síntesis, el instrumento facilitaría la reflexión sobre las estrategias o métodos que cotidianamente se utilizan para fomentar el desarrollo de habilidades de argumentación; recursos de gran valor en el ejercicio y enseñanza de las ciencias, la promoción de procesos cognitivos de orden superior y la construcción significativa de conocimientos.

\section{REFERENCIAS BIBLIOGRÁFICAS}

Brooks, C. \& Jeong, A. (2006). Effects of Pre-structuring Discussion Threads on Group Interaction and Group Performance in Computer-supported Collaborative Argumentation. Distance Education, vol. 27, n. 3, 371-390.

Cho, K. \& Jonassen, D. (2002). The Effects of Argumentation Scaffolds on Argumentation and Problem Solving. Educational Technology Research and Development, vol. 50, n. 3, 5-22.

Clark, D. \& Sampson, V. (2007). Personally-seeded Discussions to Scaffold Online Argumentation. International Journal of Science Education, vol. 29, n. 3, 253-277. , Sampson, V., Weinberger, A. \& Erkens, G. (2007). Analytic Frameworks for Assessing Dialogic Argumentation in Online Learning Environments. Educational Psychology Review, vol. 19, n. 3, 343-374.

Coll, C. (2004). Psicología de la educación y prácticas educativas mediadas por las tecnologías de la información y la comunicación. Revista Electrónica Sinéctica, n. 25, 1-24.

De Vries, E., Lund, K. \& Baker, M. (2002). Computer-mediated Epistemic Dialogue: Explanation and Argumentation as Vehicles for Understanding Scientific Notions. Journal of the Learning Sciences, vol. 11, n. 1, 63-103.

Evagorou, M. \& Avraamidou, L. (2008). Technology in Support of Argument Construction in School Science. Educational Media International, vol. 45, n. 1, 33-45.

Golanics, J. \& Nussbaum, E. (2007). Enhancing Online Collaborative Argumentation through Question Elaboration and Goal Instructions. Journal of Computer Assisted Learning, vol. 24, n. 3, 167-180.

Jeong, A. (2006). The Effects of Linguistic Qualifiers and Intensifiers on Group Interaction and Performance in Computer-supported Collaborative Argumentation. The International Review of 
Research in Open and Distributed Learning, vol. 6, n. 3, 1-18.

Jonassen, D. (2010). Learning to Solve Problems: A Handbook for Designing Problem-solving Learning Environments. New York: Routledge.

Kohlbacher, F. (2006). The Use of Qualitative Content Analysis in Case Study Research. Forum: Qualitative Social Research, vol. 7, n. 1. Recuperado de: http://www.qualitative-research.net/index. php/fqs/article/\%20view/75/153January\%202006, consultado en mayo de 2013.

Kuhn, D. \& Crowell, A. (2011). Dialogic Argumentation as a Vehicle for Developing Young Adolescents' Thinking. Psychological Science, vol. 22, n. 4, 545-552.

Goh, W., Iordanou, K. \& Shaenfield, D. (2008). Arguing on the Computer: A

Microgenetic Study of Developing Argument Skills in a Computer-supported Environment. Child Development, vol. 79, n. 5, 1310-1328.

n. $5,1245-1260$. \& Udell, W. (2003). The Development of Argument Skills. Child Development, vol. 74, (1999). The Skills of Argument. Cambridge: Cambridge University Press.

Leitão, S. (2007). La dimensión epistémica de la argumentación. En E. Kronmüller y C. Cornejo (Eds.), Ciencias de la mente: Aproximaciones desde Latinoamérica (pp. 5-32). Santiago: J.C. Sáez Editor.

(2001). Analyzing Changes in View during Argumentation: A Quest for Method. Forum: Qualitative Social Research, vol. 2, n. 3. Recuperado de: http://www.qualitative-research.net/index. php/fqs/article/view/907/1982, consultado en mayo de 2013.

. (2000). The Potential of Argument in Knowledge Building. Human Development, vol. 43 , n. 6, 332-360.

Ley, K. \& Young, D. (2001). Instructional Principles for Self-Regulation. Educational Technology Research and Development, vol. 49, n. 2, 93-103.

McMahon, M. \& Oliver, R. (2001). Promoting Self-regulated Learning in an On-line Environment. In C. Montgomerie \& J. Viteli (Eds.), Proceedings of World Conference on Educational Multimedia, Hypermedia and Telecommunications 2001 (pp. 1299-1305). Chesapeake, VA: AACE.

Maloney, J. \& Simon, S. (2006). Mapping Children's Discussions of Evidence in Science to Assess Collaboration and Argumentation. International Journal of Science Education, vol. 28, n. 15, 1817-1841.

Martí, E. (2003). Las tecnologías de la información y la comunicación. En E. Martí (Ed.), Representar el mundo externamente: La adquisición infantil de los sistemas externos de representación (pp. 213-269). Madrid: A. Machado Libros.

Mayring, P. (2004). Qualitative Content Analysis. In U. Flick, E. Von Kardorff, \& I. Steinke (Eds.), A Companion to Qualitative Research (pp. 266-269). London: Sage.

. (2000). Qualitative Content Analysis. Forum: Qualitative Social Research, vol. 1, n. 2. Recuperado de http://www.qualitative-research.net/index.php/fqs/article/view/1089, consultado en mayo de 2013.

Ministerio de Educación Nacional. (2007). Plan Nacional Decenal de Educación 2006 2016: Pacto social por la educación. Recuperado de http://www.plandecenal.edu.co/html/1726/ articles-166057_cartilla.pdf, consultado en mayo de 2013.

Montes, J. y Ochoa, S. (2006). Apropiación de las tecnologías de la información y la comunicación en cursos universitarios. Acta Colombiana de Psicología, vol. 9, n.2, 87-100.

Moore, J. \& Marra, R. (2005). A Comparative Analysis of Online Discussion Participation Protocols. Journal of Research on Technology in Education, vol. 38, n. 2, 191-212.

Munneke, L., Andriessen, J. E., Kanselaar, G. \& Kirschner, P. (2007). Supporting Interactive Argumentation: Influence of Representational Tools on Discussing a Wicked Problem. Computers in Human Behavior, vol. 23, n. 3, 1072-1088.

, Van Amelsvoort, M. \& Andriessen, J. (2003). The Role of Diagrams in Collaborative Argumentation-based Learning. International Journal of Educational Research, vol. 39, n. 1-2, 113-131. 
North, S., Coffin, C. \& Hewings, A. (2008). Using Exchange Structure Analysis to Explore Argument in Text-based Computer Conferences. International Journal of Research and Method in Education, vol. 31, n. 3, 257-276.

Onrubia, J. (2007). Las tecnologías de la información y la comunicación como instrumento de apoyo a la innovación de la docencia universitaria. Revista Interuniversitaria de Formación del Profesorado, vol. 21, n. 1, 21-36.

Perry, N. \& Winne, P. (2006). Learning from Learning Kits: Study Traces of Students' Selfregulated Engagements with Computerized Content. Educational Psychology Review, vol. 18, n. 3, 211-228.

Pozo, J. y Monereo, C. (1999). El aprendizaje estratégico. Enseñar a aprender desde el currículo. Madrid: Santillana/Aula XXI.

, Scheuer, N., Pérez, M., Mateos, M., Martín, E. y De la Cruz, M. (2006). Nuevas formas de pensar la enseñanza y el aprendizaje: Las concepciones de profesores y alumnos. Barcelona: Graó.

Sampson, V. \& Gerbino, F. (2010). Two Instructional Models that Teachers can Use to Promote and Support Scientific Argumentation in the Biology Classroom. The American Biology Teacher, vol. 72, n. 7, 427-431.

Scheuer, O., Loll, F., Pinkwart, N. \& McLaren, B. (2010). Computer-supported Argumentation: A Review of the State of the Art. International Journal of Computer-Supported Collaborative Learning, vol. 5, n. 1, 43-102.

Simon, S. \& Richardson, K. (2009). Argumentation in School Science: Breaking the Tradition of Authoritative Exposition through a Pedagogy that Promotes Discussion and Reasoning. Argumentation, vol. 23, n. 4, 469-493.

\& Maloney, J. (2007). Activities for Promoting Small-group Discussion and Argumentation. School Science Review, vol. 88, n. 324, 49-57.

, Erduran, S. \& Osborne, J. (2006). Learning to Teach Argumentation: Research and Development in the Science Classroom. International Journal of Science Education, vol. 28, n. 2-3, 235-260.

Stark, R., Puhl, T. \& Krause, U. (2009). Improving Scientific Argumentation Skills by a Problem-based Learning Environment: Effects of an Elaboration Tool and Relevance of Student Characteristics. Evaluation and Research in Education, vol. 22, n. 1, 51-68.

Stegmann, K., Weinberger, A. \& Fischer, F. (2007). Facilitating Argumentative Knowledge Construction with Computer-supported Collaboration Scripts. International Journal of ComputerSupported Collaborative Learning, vol. 2, n. 4, 421-447.

Valencia, M., Duarte, J. y Caicedo, A. (2011). Metas académicas y aprendizaje autorregulado en la preparación de exámenes en estudiantes universitarios. Manuscrito inédito, Carrera de Psicología, Pontificia Universidad Javeriana, Cali, Colombia.

Van Amelsvoort, M., Andriessen, J. \& Kanselaar, G. (2007). Representational Tools in Computersupported Collaborative Argumentation-based Learning: How Dyads Work with Constructed and Inspected Argumentative Diagrams. Journal of the Learning Sciences, vol. 16, n. 4, 485-521.

Winne, P. (2001). Self-regulated Learning Viewed from Models of Information Processing. In B. Zimmerman \& D. Schunk (Eds.), Self-Regulated Learning and Academic Achievement: Theoretical perspectives (pp.153-189). MahWah, NJ: Lawrence Erlbaum Associates.

\& Hadwin, A. (1998). Studying as Self-regulated Learning. In D. Hacker, J. Dunlosky \& A. Graesser (Eds.), Metacognition into Theory and Practice (pp. 277-304). New Jersey: Lawrence Erlbaum Associates.

Winters, F., Greene, J. \& Costich, C. (2008). Self-regulation of Learning within Computer-Based Learning Environments: A Critical Analysis. Educational Psychology Review, vol. 20, n. 4, 429-444. 